\section{Quels critères pour l'examen de spécialiste?}

Ch. Hänggeli, responsable du département de formation postgraduée et continue
Font exception, les candidats remplissant l'une des deux conditions suivantes:

- Les candidats ayant déjà participé à un examen de spécialiste (entièrement ou en partie) avant la fin 1998 ne doivent pas passer d'autre examen.

- Les candidats ayant terminé leur formation postgraduée jusqu'au 31 décembre 2000 ne doivent attester, pour l'obtention du titre, que leur participation à l'examen. Attention: toutes les conditions doivent avoir été remplies jusqu'à la fin de l'an 2000 (y compris l'examen de spécialiste complet, sauf si la société de discipline médicale a refusé la participation à la $2^{\mathrm{e}}$ partie de l'examen en raison d'un résultat insuffisant à la $1^{\text {re }}$ partie)! de l'examen de spécialiste pour les spécialités suivantes: médecine du travail, endocrinologie-diabétologie, hématologie, médecine intensive, oncologie médicale, oto-rhino-laryngologie (sans la chirurgie cervico-faciale), cardiologie pédiatrique, psychiatrie et psychothérapie, médecine légale, rhumatologie et médecine tropicale.

Le Comité central a mis en vigueur au $1^{\mathrm{er}}$ janvier 2001 la clause éliminatoire de l'examen de spécialiste pour onze autres spécialités. Ainsi, la réussite de l'examen de spécialiste est désormais exigée pour la moitié des titres FMH, sous réserve des dispositions transitoires applicables.

Nous publions ci-après un aperçu actuel des disciplines où la réussite de l'examen de spécialiste est obligatoire et de celles où seule la participation à l'examen est requise. Il convient de différencier 5 catégories:

\section{Entrée en vigueur au $1^{\text {er }}$ janvier 1986}

Le titre de spécialiste n'est attribué depuis plus de dix ans qu'après réussite de l'examen de spécialiste dans les disciplines suivantes:

- anesthésiologie,

- chirurgie pédiatrique,

- radiologie,

- neurochirurgie.

\section{Entrée en vigueur au $1^{\text {er }}$ janvier 1999}

La réussite de l'examen de spécialiste - sous réserve des exceptions énumérées ci-après - est également exigée pour le titre de spécialiste dans les disciplines suivantes, depuis le $1^{\text {er }}$ janvier 1999:

- chirurgie,

- gynécologie et obstétrique,

- médecine interne,

- cardiologie,

- pathologie.
L'examen de base en chirurgie pour l'obtention du titre de spécialiste en chirurgie ( $1^{\text {re }}$ partie de l'examen de spécialiste) constitue un cas particulier:

La participation à la $1^{\text {re }}$ partie de l'examen de spécialiste avant la fin de 1998 ne dispense pas de la $2^{\mathrm{e}}$ partie. Les candidats ayant terminé leur formation postgraduée en chirurgie générale jusqu'au 31 décembre 2000 doivent présenter une attestation de leur participation à la $2^{\mathrm{e}}$ partie de l'examen afin d'obtenir le titre de spécialiste en chirurgie. Les candidats n'ayant pas obtenu cette attestation (parce qu'ils n'ont pas réussi l'examen de base) et qui, de ce fait, n'ont pas été admis à la $2^{\mathrm{e}}$ partie de l'examen, ne doivent attester que la participation à l'examen de base. Les candidats ne terminant leur formation postgraduée qu'après le 31 décembre 2000 doivent en tout cas attester la réussite de l'examen de spécialiste (examen de base compris).

\section{Entrée en vigueur au $1^{\mathrm{er}}$ janvier $\mathbf{2 0 0 0}$}

La réussite de l'examen de spécialiste - sous réserve des exceptions énumérées ci-après - est également exigée pour le titre de spécialiste dans les disciplines suivantes, depuis le $1^{\text {er }}$ janvier 2000:

- médecine générale,

- gastroentérologie.

Font exception, les candidats remplissant l'une des deux conditions suivantes:

- Les candidats ayant déjà participé à un examen de spécialiste (entièrement ou en partie) avant la fin 1999 ne doivent pas passer d'autre examen.

- Les candidats terminant leur formation postgraduée d'ici le 31 décembre 2001 ne doivent attester, pour l'obtention du titre, que leur participation à l'examen. Attention: toutes les conditions doivent être remplies d'ici la fin de l'an 2001 (y compris l'examen de spécialiste complet, sauf si la société de discipline médicale refuse la participation à la $2^{\mathrm{e}}$ partie de l'examen en raison d'un résultat insuffisant à la $1^{\text {re }}$ partie)! 


\begin{tabular}{|c|c|c|c|c|c|}
\hline \multicolumn{6}{|c|}{ Dois-je réussir l'examen de spécialiste? } \\
\hline & &  & 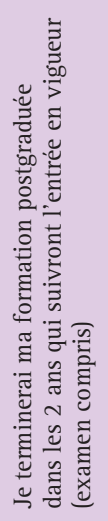 & 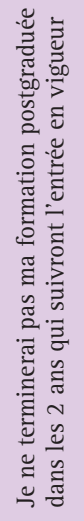 & 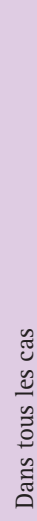 \\
\hline Médecine générale & 01.01 .2000 & $\mathrm{P}$ & $\mathrm{P}$ & $\mathrm{R}$ & - \\
\hline Anesthésiologie & 02.04 .1986 & - & - & - & $\mathrm{R}$ \\
\hline Médecine du travail & 01.01 .2001 & $\mathrm{P}$ & $\mathrm{P}$ & $\mathrm{R}$ & - \\
\hline Chirurgie (y c. examen de base) & 01.01 .1999 & $P$ & $\mathrm{P}$ & $\mathrm{R}$ & - \\
\hline Endocrinologie-diabétologie & 01.01 .2001 & $P$ & $P$ & $\mathrm{R}$ & - \\
\hline Gastroentérologie & 01.01 .2000 & $\mathrm{P}$ & $\mathrm{P}$ & $\mathrm{R}$ & - \\
\hline Gynécologie et obstétrique & 01.01 .1999 & $\mathrm{P}$ & $\mathrm{P}$ & $\mathrm{R}$ & - \\
\hline Hématologie & 01.01 .2001 & $\mathrm{P}$ & $\mathrm{P}$ & $\mathrm{R}$ & - \\
\hline Médecine interne & 01.01 .1999 & $\mathrm{P}$ & $\mathrm{P}$ & $\mathrm{R}$ & - \\
\hline Médecine intensive & 01.01 .2001 & $P$ & $P$ & $\mathrm{R}$ & - \\
\hline Cardiologie & 01.01 .1999 & $\mathrm{P}$ & $\mathrm{P}$ & $\mathrm{R}$ & - \\
\hline Chirurgie pédiatrique & 02.04 .1986 & - & - & - & $\mathrm{R}$ \\
\hline Oncologie médicale & 01.01 .2001 & $P$ & $\mathrm{P}$ & $\mathrm{R}$ & - \\
\hline Neurochirurgie & 02.04 .1986 & - & - & - & $\mathrm{R}$ \\
\hline $\begin{array}{l}\text { ORL (sans chirurgie } \\
\text { cervico-faciale) }\end{array}$ & 01.01 .2001 & $\mathrm{P}$ & $\mathrm{P}$ & $\mathrm{R}$ & - \\
\hline Pathologie & 01.01 .1999 & $\mathrm{P}$ & $\mathrm{P}$ & $\mathrm{R}$ & - \\
\hline Cardiologie pédiatrique & 01.01 .2001 & $\mathrm{P}$ & $\mathrm{P}$ & $\mathrm{R}$ & - \\
\hline Psychiatrie et psychothérapie & 01.01 .2001 & $\mathrm{P}$ & $\mathrm{P}$ & $\mathrm{R}$ & - \\
\hline Radiologie & 02.04 .1986 & - & - & - & $\mathrm{R}$ \\
\hline Médecine légale & 01.01 .2001 & $\mathrm{P}$ & $\mathrm{P}$ & $\mathrm{R}$ & - \\
\hline Rhumatologie & 01.01 .2001 & $\mathrm{P}$ & $\mathrm{P}$ & $\mathrm{R}$ & - \\
\hline Médecine tropicale & 01.01 .2001 & $\mathrm{P}$ & $\mathrm{P}$ & $\mathrm{R}$ & - \\
\hline tous les autres domaines & & - & - & - & $\mathrm{P}$ \\
\hline $\mathrm{R}=$ réussite, $\mathrm{P}=$ participation & & & & & \\
\hline
\end{tabular}

\section{Entrée en vigueur au $1^{\text {er }}$ janvier 2001}

La réussite de l'examen de spécialiste - sous réserve des exceptions énumérées ci-après - est également exigée pour le titre de spécialiste dans les disciplines suivantes, depuis le $1^{\text {er }}$ janvier 2001:

- médecine du travail,

- endocrinologie-diabétologie,

- hématologie,

- médecine intensive,

- oncologie médicale,

- oto-rhino-laryngologie

(sans chirurgie cervico-faciale),

- psychiatrie et psychothérapie,

- médecine légale,

- rhumatologie,

- médecine tropicale,

- cardiologie pédiatrique (formation approfondie).

Font exception, les candidats remplissant une des deux conditions suivantes:

- Les candidats ayant déjà participé à un examen de spécialiste (entièrement ou en partie) avant la fin 2000 ne devront pas passer d'autre examen.

- Les candidats terminant leur formation postgraduée d'ici le 31 décembre 2002 ne doivent attester, pour l'obtention du titre, que leur participation à l'examen. Attention: toutes les conditions doivent être remplies d'ici la fin de l'an 2002 (y compris l'examen de spécialiste complet, sauf si la société de discipline médicale refuse la participation à la $2^{\mathrm{e}}$ partie de l'examen en raison d'un résultat insuffisant à la $1^{\text {re }}$ partie)!

\section{Pas encore d'examen éliminatoire}

Dans toutes les autres disciplines, seule l'attestation de participation à l'examen de spécialiste est pour le moment nécessaire. La réussite de l'examen de spécialiste sera exigée au plus tôt à partir du $1^{\mathrm{er}}$ janvier 2002. A l'instar des disciplines précitées, un délai transitoire de deux ans sera applicable.

La documentation relative à la formation postgraduée (avec les dates actuelles d'examens) peut être consultée sur internet à l'adresse suivante: www.fmh.ch. Les dates des examens sont aussi publiées dans le Bulletin des médecins suisses. Pour de plus amples renseignements, veuillez contacter le département de formation postgraduée et continue de la FMH. Adresse: Case postale 293, Elfenstrasse 18, 3000 Berne 16, tél. 03135911 11, fax 03135911 12, e-mail: fmhdipl@hin.ch. 